\title{
LAS MNEMOTECNICAS EN LA ENSEÑANZA Y APRENDIZAJE DE ELE EN EL PROYECTO GUAMÁ BILINGÜE
}

\author{
Daniele Mendonça de PAULA CHAVES ${ }^{1}$ \\ Universidade Federal do Pará - Campus Castanhal \\ Jacqueline Augusta LEITE DE LIMA ${ }^{2}$ \\ Universidade Federal do Pará
}

\begin{abstract}
RESUMO: Nos dedicamos a apresentar como as técnicas de memória auxiliam no processo de ensino e aprendizagem do espanhol como língua estrangeira para os alunos do projeto Guamá Bilíngue. Desde a elaboração do material didático, seguida por sua aplicação em sala de aula, até as respostas dos alunos, cada passo onde é possível verificar as técnicas de memória, como a repetição de estruturas conhecidas, o uso de vocabulário próximo ao português, a relação com os conteúdos que estão presentes na vida do aluno que são utilizados na didática do projeto e na retórica dos professores como ferramentas para facilitar o processo de aprendizagem de E/LE. Segundo Taylor (1987) y Yates (2007), ao analisar outros estudiosos, as técnicas de memória são muito influentes na formação social e ideológica desde seus usos na retórica religiosa até na colonização do novo mundo. Para o desenvolvimento desta pesquisa o trabalho teve um carácter documental e bibliográfico, analisando não somente o material didático utilizado em sala, como também anotações dos professores do projeto. O embasamento teórico está centrado em estudos da teoria de aprendizagem significativo (Ausubel apud Moreira e Mansini, 1982); as técnicas de memória (Taylor, 1987 y Yates, 2007) e aprendizagem de LE (Molina, 2000). Concluímos mediante análises bibliográficos e experiências vividas nas aulas do projeto que os alunos utilizam consciente e inconscientemente técnicas de memorias para adquirir suas competências en língua estrangeira além de passarem por uma mudança crítica no meio social, que é um dos objetivos do projeto Guamá Bilíngue.
\end{abstract}

PALAVRA-CHAVE: Mnemotécnicas, Ensino/Aprendizagem, Guamá Bilíngue.

ABSTRACT: We are dedicated to presenting how memory techniques help in the process of teaching and learning Spanish as a foreign language for students of the Guamá Bilingue project. From the preparation of the didactic material, followed by its application in the classroom, to the students' answers, each step where it is possible to verify the memory techniques, such as the repetition of known structures, the use of vocabulary close to portuguese, the relation with the contents that are present in the life of the student that are used in the didactics of the project and in the rhetoric of the teachers as tools to facilitate the learning process of E / LE. According to Taylor (1987) y Yates (2007), when analyzing other scholars, memory techniques are very influential in social and ideological formation from their use in religious rhetoric to the colonization of the new world. For the development of this research the work had a documentary and bibliographic character, analyzing not only the didactic material used in the room, but also notes of the teachers of the project. Theoretical background is centered on studies of significant learning theory (Ausubel apud Moreira and Mansini, 1982); Memory techniques (Taylor, 1987 and Yates, 2007) and LE learning (Molina, 2000). We conclude through bibliographical analyzes and experiences lived in the classes of the project that students consciously and unconsciously use memory techniques to

\footnotetext{
${ }^{1}$ Professora especialista em lingua portuguesa. E-mail: danchaves_8@yahoo.com.br

${ }^{2}$ Graduanda do curso de licenciatura letras em lingua española. E-mail: jacquelineleitedelima@gmail.com
} 
acquire their foreign language skills and to undergo a critical change in the social environment, which is one of the objectives of the Guamá Bilingue project.

KEYWORDS: Mnemotecnicas, Teaching and learning, Guamá Bilingue

\section{Introducción}

Dentro de la formación académica en licenciatura de letras/lengua español, las dos autoras tuvieron experiencias semejantes en periodos próximos, pasaron por experiencias en dos áreas: la primera, teniendo experiencias en el proyecto social Guamá Bilingüe, lo que las llevo a encontrar afinidades en las investigaciones académicas direccionadas al uso de los textos y de la literatura como herramienta en la enseñanza del español como lengua extranjera (E/LE), y la segunda, dentro de estudios científicos en el ámbito de la literatura y rasgos específicos dentro de algunas obras literarias del contexto hispano, encontrándose otra vez en la similitud de los contextos estudiados que involucraban la medievalidad y los textos del nuevo mundo.

Las experiencias semejantes y compartidas por las autoras les despertó un interés mutuo en la área de la didactica y la literatura buscando conectar los rasgo, las especifidades, los temas y las funciones de los textos literarios con las metodologías y actuaciones en clases de lengua extranjera, considerando principalmente el contexto de enseñanza del proyecto social Guamá Bilingüe en el cual ambas actúan en distintas estancias, una como parte del cuerpo técnico/pedagógico y otra actuando dentro del aula como docente/becaria.

A lo largo de esta sociedad se destacó dos temas pesquisados en áreas diferentes que parecían no encajarse, la investigación enfocada en las técnicas y memorias presentes en los escritos cronísticos históricos/literarios del descubrimiento de las Américas y el interés en reconocer las técnicas de enseñanza y aprendizaje dentro de todo el contexto que abarca el proyecto Guamá Bilingüe. Estas dos investigaciones parecían tan lejanas en sus características específicas que su unión parecía improbable.

Sin embargo, lo improbable se puso probable al desarrollar unas lecturas y debates acerca del uso de los textos literarios en la enseñanza de español, específicamente al leer la cita que Pardo (2008) hace acerca de Molina (2000) afirmando que "el lector tiende a imitar la expresión del autor, capta y memoriza inconscientemente el léxico, (...) haciéndolos suyos, y utilizándolos después en sus futuras expresiones". Fue así que las ideas se encontraron y se puso clara, prácticamente obvia, una relación, científicamente comprobada y que, ya habíamos, inconscientemente, observado entre las técnicas y memorias y el uso de los textos, tal como la posibilidad de las mnemotécnicas funcionaren como herramientas en el momento de aprenderse una nueva lengua.

Partiendo de esto, surgió el cuestionamiento de como podría ser comprobada la existencia del uso las técnicas de memorias, identificadas en los estudios del proyecto de investigación $O$ lugar do medieval e da memória em Gonzalo Fernández de Oviedo y Valdés, dentro de la metodología del proyecto de enseñanza, investigación y extensión Guamá Bilingüe considerando, principalmente, el uso que el mismo hace de los textos.

En la intención de contestar este cuestionamiento, fue observado que la metodología del proyecto Guamá Bilingüe era de suma importancia en el aprendizaje de los alumnos, pues facilitaba la asimilación de vocablos, estructuras, expresiones y tornaba más fácil para el alumno aprender a utilizar la nueva lengua teniendo contacto con la misma en uso, lo que es hecho, en el proyecto, a través de las clases impartidas en español desde el primer momento y, 
principalmente, por el uso de los textos como apoyo, textos de diversos géneros, que hacen con que el alumno aprenda una nueva lengua, conozca nuevas culturas y pase por un cambio comportamental relacionado a la filosofía moral y social instruida por el proyecto.

Las conclusiones a cerca del proyecto Guamá Bilingüe, se acercaron a las conclusiones de las investigaciones hecha en el ámbito de la memoria, en el proyecto de investigación $O$ lugar do medieval e da memória em Gonzalo Fernández de Oviedo y Valdés, pues en ello analizamos la función de la memoria dentro de los relatos ficticios/históricos así como el inicio del uso de las técnicas de memoria en la retórica religiosa.

Fue una sorpresa percibir por ende que el lugar de la memoria era hacer con que el lector/oyente aprendiese algo nuevo, conociese nuevos hábitos y culturas y sufriese influencia en su formación ideológica, intelectual y social a través de técnicas de memoria. Funciones que encontramos, también, dentro de las intenciones y resultados del proprio proyecto social Guama Bilingüe, como mencionado anteriormente.

Bajo estas semejanzas entre as observaciones y conclusiones de ambas las áreas de investigación fue que empezamos a trabajar con teorías y marcos bibliográficas abordando la existencia de tal relación entre los textos y el uso de las técnicas de memoria, lo que fue una tarea, no tan simples, de organizar y madurar las ideas. Y es exactamente esto que nos proponemos a mostrar en este trabajo, mostrar como que las técnicas de memorias son utilizados como herramienta en la enseñanza/aprendizaje de E/LE, principalmente cuando esta enseñanza posee un enfoque social como en el proyecto Guamá Bilingüe.

\section{Las primeras hipótesis}

Estudios anteriores nos permitieron percibir que dentro del proceso de enseñanza y aprendizaje hacemos uso eminente de la memoria como un simples reflejo mental, juntamente, utilizamos también las técnicas de memoria, pues necesitamos de actividades que trabajen con el reconocimiento, registro, organización, integración y retención de las informaciones que recibimos en el procedimiento de aprendizaje, de la misma manera que utilizamos estas técnicas de memoria en el proceso de enseñanza, no importando cual asignatura.

También es sabido que en ambos los procesos, de aprendizaje y enseñanza, es necesario hacer el uso de textos, sea oral o escritos, pues estos textos funcionan como herramientas, introduciendo, exponiendo o explicando un determinado tema que dentro del proceso de aprendizaje y lectura el alumno, tal como el propio profesor en el proceso de elaboración y ejecución de la enseñanza, necesita recorrer a la memoria para realizar una correlación de los hechos aprendidos con sus vivencias, tornando posible reflexionar y reformular las ideas con el propósito de generar una producción escrita y/o oral sobre determinado tema.

Partiendo de estos dos saberes, es ya posible afirmar la relación que existe entre la memoria y los textos utilizados en el proceso de enseñanza/aprendizaje, lo que nos hace pensar a cerca de las técnicas de memoria dentro de estos procesos en la área de enseñanza que nos interesa, o sea, el Español como Lengua Extranjera (E/LE), desde una perspectiva y metodología especifica del proyecto social de enseñanza, investigación y extensión Guamá Bilingüe, en el cual las autora tuvieron participación en diverso ámbitos permitiendo así un análisis más amplio de las técnicas de memoria desde la elaboración y aplicación del material, en la planificación y ejecución de las clases, percepción y practica de la pedagogía del proyecto, así 
como una visión de investigadoras analizando el comportamiento de los alumnos y profesores.

Dentro de la metodología del proyecto se incluí algunos métodos que influyen en el aprendizaje, como por ejemplo el uso de diversos tipos de texto como aporte y herramientas dentro del material didácticos. Es posible, también, encontrar las prácticas de andamiaje de Vygotsky (1989) dentro del aula, a partir del momento que los profesores son alumnos de la carrera y poseen algún nivel más que los alumnos cuanto el aprendizaje de la lengua extranjera. El aprendizaje significativa de Ausubel (1983), considerando Ausubel apud Moreira \& Mansini (1982), está presente cuando se explota la zona de transparencia del español con el portugués y al hacer uso de la didáctica asociativa y no traductora. También toma provecho del uso del discurso de autoridad del profesor dentro del proceso de formación social, visto que las intenciones del proyecto son volcadas para la formación ciudadana de alumnos en riesgo social.

A lo largo de algunas investigaciones podemos percibir que, basado en estudios bibliográficos, es posible reconocer el uso de técnicas de memorias (mnemotécnicas) dentro de la enseñanza del español, principalmente, cuando esta está pautado por las mismas directrices descritas dentro del proyecto Guamá Bilingüe y sus ramificaciones (Proyecto Língua, cultura e cidadania y Proyecto Formação cidadã em E/LE). Creemos que estas mnemotécnicas son usadas dentro do proyecto, en la elaboración metodológica y en el material, en la metodología aplicada en clase y como herramienta para trabajar la formación ciudadana, sin embargo, en su grande mayoría son utilizadas de manera inconsciente, tanto por los alumnos como por los propios profesores.

\section{Marco teórico y metodología}

Las técnicas de memoria son utilizadas en diversos ámbitos, en esta investigación pretendemos trabajar con las técnicas de memoria en tres áreas: dentro de la retórica, en el discurso de autoridad del profesor que es utilizado como la base de la metodología del proyecto social Guamá Bilingüe; en la producción escrita, dentro de los textos utilizados como herramientas de apoyo en el material del proyecto; así como técnicas de aprendizaje, usadas por los alumnos en el momento de asociar una palabra en español a su zona de transparencia en portugués o al aprender a utilizar expresiones partiendo de un referencial, sean ellos escritos u orales.

Para que podamos analizar la memoria en estos diversos, consideramos las técnicas de memoria citadas y definidas por Yates (2007), en el libro el Arte da memória, donde se menciona y explica todos los tratados sobre memoria, que nos trae la primera idea del proceso de asociación, como una técnica de memoria, partiendo del tratado de Ad Herenmium y perpetuando tras la "evolución" de las técnicas por medio de asociación de loci e imagens, lo cual podemos traer para la práctica en el aula cuando ocurre la asociación hecha entres el significado y significante de Saussure (1996), o en la técnica de trabajarse la producción oral a través del constante ejercicio de la comprensión auditiva, también según Saussure (2002).

Cuanto a las técnicas de memoria relacionadas a la enseñanza, principalmente volcada a la práctica de la lectura, nos dedicamos a Rossi (2010) que no solamente asocia las mnemotécnicas dentro de la enseñanza a los textos como hace referencia al uso de textos como bases concretas de apoyo para la asociación de imágenes, hechos y temas con el 
propósito de provocar un cambio comportamental, como el cambio social que se tiene como propuesta y objetivo en el enfoque del proyecto Guamá Bilingüe.

De esta manera, Rossi (2010) afirma que “Ás imagens havia sido consignada uma dupla tarefa: fixar conceitos na memória, agir sobre a vontade e, em consequência, modificar os comportamentos", siguiendo así la intención por tras del proyecto social, que propone a través de la enseñanza del español enseñar un nuevo léxico, que optamos por llamar de "imagem", para fijar un concepto en la memoria del aluno (des) construyendo pensamientos existentes y criados pelo medio en que estos alumnos viven y en consecuencia de este proceso provocar el cambio comportamental de estos alumnos dentro de los principios sociales.

Semejante a Rossi (2010), estudiamos también como las técnicas de memoria fueran utilizadas, con la misma finalidad del proyecto, o sea volcada al cambio comportamental y enseñanza, en el inicio de la colonización del nuevo mundo, donde en principio se trabajó con la tentativa de una enseñanza doctrinaria, volcado al trabajar con nuevas culturas y lenguas, teniendo el profesor y el aprendiz diferentes signos maternos de comunicación, pero teniendo éxito en el proceso de enseñanza/aprendizaje, como relata Taylor (1987) al decir que en sus estudios descubrió que los jesuitas, mas específicamente Frei Diego, utilizaba las técnicas de memoria para repasar a los nativos las culturas, costumbres y lengua de los conquistadores.

Ya que tratamos de un ambiente especifico de estudios, es importante que consideremos las particularidades del proyecto social Guamá Bilingüe, desde las perspectivas generales sobre la ideología, formación y aplicación del proyecto hechas por Paiva (2012), nos hemos profundizado en aspectos de la metodología como el proceso de aprendizaje significativo de Ausubel (1983), donde ya hay la presencia de algunas técnicas de memoria, así como el proceso enseñanza por andamiaje de Vygotsky (1989) y la metodología apoyada en el uso de textos.

Para analizar y demonstrar la relevancia y relación entre as mnemotécnicas y los textos dentro del proyecto pasamos directamente a los estudios volcados para el uso de textos en la enseñanza de E/LE con Santos (2004) y Nascimento (2014), tal como Pardo (2008) y Molina (2000) que tratan el uso de textos como auxiliadores en la memorización de léxicos y estructuras, así como Lima (2014) que trata, específicamente de la función del usos de textos dentro del proyecto Guamá Bilingüe, además de utilizar como aporte los informes hecho por los profesores/becarios o voluntarios del proyecto en el intento de identificar el uso de las técnicas por los profesores como también por los alumnos.

\section{Las mnemotécnicas actuando en clase}

El proyecto Guamá Bilingüe existe en la $\mathrm{UFPA}^{3}$ desde el año 2010, su intención social se mezcla con el auxilio en la formación, a través de la práctica de docencia y la elaboración de materiales didácticos para los estudiantes de la carrera de letras/español que participan del proyecto. Este material elaborado, posee dimensiones específicas que siguen un padrón que visa siempre utilizar un texto como aporte, un tema social para debatir en clase y un apartado de gramática junto al uso de léxicos que sean de fácil comprensión para los jóvenes alumnos del proyecto, que nunca tuvieron, en su mayoría, un contacto con la lengua española.

\footnotetext{
${ }^{3}$ Universidade Federal do Pará
} 
Además de preocuparse con que el material posea un léxico accesible, el entrenamiento de los futuros profesores del proyecto y la actuación de los mismos en clase está pautada por la teoría del aprendizaje significativo de Ausubel (1983), o sea, para el proyecto, el aprendizaje debe hacer con que el estudiante relacione la información nueva con la que ya posee, reajustando y reconstruyendo las informaciones en este proceso. Este hecho de trabajar con informaciones conocidas para aclarar mejor el desconocido está para nosotros relacionado a una técnica de memoria que consiste, según Rossi (2010), en el hecho de que recordar o reconocer es algo que solo se puede pasar si hay una vivencia y conocimiento previo de los hechos, es decir, el alumno utiliza esta relación de transparencia que hay entre el español y el portugués únicamente por poseer un conocimiento de este léxico o expresión en su lengua materna, y lo hace de manera consciente o inconsciente.

En el ámbito de las mnemotécnicas utilizadas en la enseñanza/aprendizaje y haciendo una comparación a las metodologías y didácticas del proyecto Guama Bilingüe, también podemos traer el hecho de que en el periodo del descubrimiento de las Américas los colonizadores utilizaron técnicas de memoria para doctrinar, enseñar algo de la cultura europea para los indios, como lo afirma Taylor (1987), así como apunta Yates (2007), que las mnemotécnicas poseen una función histórica al utilizar los marcos e imagines conocidas para mejor explicar o relacionar la nueva información.

De cierta manera es lo que hace la dinámica del proyecto Guamá Bilingüe al utilizar un contexto y temas que son de conocimiento de los aprendientes y que están presente en los textos para intentar desarrollar un debate que lleve el alumno a reflexionar su cultura y sus actitudes frente a la nueva cultura y distintas actitudes presentes en los textos con la intención final de hacer un cambio en su comportamiento. Esta función social, que posee el proyecto Guamá Bilingüe y al cual se aplican las mnemotécnicas, es algo que muchos estudiosos apuntan como una de las funciones del uso de los textos literarios en la enseñanza de E/LE, entre ellos Nascimento (2004) que dice:

Tengo muy presente la importancia de nuestra actividad en la formación de ciudadanos, sean cuales sean nuestros alumnos, teniendo la lengua española y todo lo que le concierne como instrumento de trabajo. Es más: creo firmemente que el texto literario es un instrumento fundamental en esa transformación. (NASCIMENTO, 2014 p. 161)

En este mismo texto de donde sacamos la citación anterior, Nascimento (2014) afirma que la literatura no encontró un espacio en las clases de lengua extranjera porque es trabajada de una manera equivocada, como explica Santos (2004) cuando afirma que los textos literarios son utilizados en clase como cualquier otro material, en general lo que se explota son la práctica de los aspectos gramaticales, y este es uno de los diferenciales que posee el proyecto Guamá Bilingüe al trabajar con los textos literarios. Como nos cuenta Lima (2014), al hablar de la diferencia del uso de los textos, según las escuelas públicas brasileñas y el proyecto:

Muchos manuales didácticos trabajan los textos literarios de forma limitada, los ejercicios poco favorecen a la creatividad del alumno, los textos están marginados como simples apoyo a la enseñanza y aprendizaje de la gramática, sin explotar su verdadero valor. [...] Frente este análisis es que el material del proyecto Guamá Bilingüe es basado en el uso de un léxico con grande proximidad al portugués, para facilitar el aprendizaje pues esta 
semejanza: permite establecer, desde las primeras clases, un ambiente de confianza para los alumnos [...] (LIMA, 2014 p. 696)

De este modo que los materiales del proyecto son elegidos llevando en consideración los asuntos que involucran, el alumno y su sociedad. Así el estudiante se identifica en los contextos mencionados, pues están cerca de su realidad, y con eso él se percibe como ciudadano. González (2011) afirma la importancia de aproximar los textos del medio en el cual vive el alumno y sus condiciones sociales en el momento de seleccionar los materiales que van a ser utilizados en clase. A través de estos textos, con temas conocidos por el estudiante se intenta llegar, a través de la educación, a los valores morales, teniendo en el aula un espacio para desarrollar la conciencia crítica, los valores estéticos e históricos.

Otro punto importante que se puede notar en la influencia de los textos y del uso de las mnemotécnicas en la enseñanza/aprendizaje es como los textos, según Pardo (2008), auxilian en la memorización de léxicos, expresiones y aspectos específicos de la lengua en que los textos están escritos. Así como Molina (2000) afirma que el lector imita, memoriza y capta las expresiones del autor, sus léxicos, construcciones y recursos expresivos tomando para si lo que encuentran en los textos, tal como muchos alumnos del proyecto que según relatos de los informes de los profesores utilizan en sus producciones escritas y orales las expresiones y vocablos más utilizados en clase.

Sobre esto, Pardo (2008) afirma que "Una ventaja más es que los textos literarios son input comprensibles para la adquisición de estructuras de la lengua meta". Eso notamos en los alumnos y en afirmaciones de los becarios que actúan como profesores en el proyectos, los alumnos inconscientes o no demonstrar aprender muy más fácilmente como utilizar las expresiones y léxicos que a menudo son utilizada en clase sea en el habla de los profesores o en los textos.

\section{Conclusión}

En síntesis afirmamos que se puede notar como el proyecto Guamá Bilingüe utiliza los textos literarios y las mnemotécnicas al manejar una metodología y didactica que acerca los temas y contenidos de la realidad de los alumnos, debatiendo en clase temas que estén presentes en el contexto mundial para poder hacer relación con los rasgos hispanos y maternos de los alumnos usando estrategias de léxicos y estructuras repetidas, cercanos a la lengua materna y un discurso que intenta enseñar los alumnos a ejercer una perspectiva de comportamiento social que tienen o no conocimiento.

Es sabido que este uso de los textos literarios y de las técnicas de memorias actúan en el ámbito de la enseñanza desde mucho, sin embargo su utilización ni siempre es consciente de por parte de los alumnos y de los propios profesores que muchas veces no reconocen que hay una manera de utilizar la literatura para fines que no sean solamente reconocimiento gramatical o análisis de estructuras.

Es importante que se abandone la idea de complejidad que existe en el uso de la literatura y se cuestione de qué manera podemos utilizarla en clase. Pese que hay infinitas posibilidades de trabajar con la literatura en clase, la intención de formación ciudadana a través de los textos literarios, actuando en un contexto de enseñanza social es lo que ponemos como algo 
fundamental, pues a lo largo de las análisis que hicimos de la historia y de los estudios de la arte de la memoria y del uso de los textos en la enseñanza la intención de "civilizar".

Como dijo Manuel, el profesor personaje, en la página 89 y 90 del libro de Luis Landero: Entre líneas, "pocas cosas hay tan necesarias hoy como enseñar historia, filosofía y literatura. Sin ellas no conseguimos civilizar... nadie sabe qué otra cosa podría salvarlo". Pensando en esto el proyecto Guamá Bilingüe tiene en su didáctica y metodología, en los rasgos de mnemotécnicas y en los textos literarios las herramientas para rescatar o salvar a estos jóvenes en riesgo social que hacen parte del proyecto.

\section{Referencias}

AUSUBEL, N-H. Psicología Educativa: Un punto de vista cognoscitivo. 1983. $2^{\circ}$ Ed. TRILLAS, México.

GONZÁLEZ, M. En torno al sentido de la enseñanza de las literaturas de lengua española en la Universidad Brasileña. In: CORDEIRO et al (org.). Hispanismo no brasil: reflexões e sentidos em construção. São Carlos: Pedro \& Joao Editores. São Paulo, 2014, p. 175-190

LIMA, J. A. L. de; PAIVA, R, de C. El uso de los textos como base y apoyo en la enseñanza del e/le. In: TIRLONI, Larissa Paula; MARINHO, Marcelo; CORTEZ, Mariana (Org.). Encontro Internacional de Letras, ed. 8. JORNADAS LATINOAMERICANAS DE LINGUAGENS E CULTURA, ed. 1. JORNADINHAS DE LITERATURA INFANTIL E ENSINO, ed. 1. 2014, Foz do Iguaçu/PR. Unioeste/Unila, 2014, p. 695-699. Anais.

MOLINA, J. L. La literatura infantil y juvenil en la enseñanza-aprendizaje de la lengua y literatura. Instituto de Estudios Almerienses. Almería, 2000.

MOREIRA, M A. \& MASINI, E. F. S. Aprendizagem significativa: A teoria de David Ausubel. São Paulo: Moraes. 1982

NASCIMENTO, M. B. B. La literatura de lengua española en los cursos fundamental y medio: ¿eso importa? In: CORDEIRO et al (org.). Hispanismo no brasil:reflexões e sentidos em construção. São Carlos: Pedro \& Joao Editores. São Paulo, 2014, p. 155-173.

PAIVA, R. de C. Guamá Bilingüe: la enseñanza de español multicultural para alumnos en situación de riesgo social. In: COLLOQUE « LES LANGUES LATINES ET L'INTERCULTURALITE. Moscou: Editora Universitaria, 2012, p. 106-110.

PARDO FORTES MONTSERRAT, N, 2008. «Los textos literarios en el aula de E/LE»www.educacion.gob.es/.../redele/...RedEle/.../201. Accedido en 28 de Octubre de 2016.

ROSSI, P. O passado, a memória, o esquecimento: seis ensaios da história das ideias / Paolo Rossi; tradução Nilson Moulin. - São Paulo: Editora UNESP, 2010.

SANTOS, A. C El texto literario: su importancia en la enseñanza y aprendizaje de E/LE. In: BENÍTEZ PÉREZ, Pedro; ROMERO GUILLEMAS, Raquel (Coords.). Actas del I Simposio de Didáctica Español para Extranjeros: Teoría y Práctica. Rio de Janeiro: Associação Hispano Brasileira Instituto Cervantes, 2004. p. 82-93. 
SAUSSURE, F de. Curso de Linguística Geral. Organizado por Charles Bally e Albert Sechehaye. Tradução de Antônio Chelini, José Paulo Paes e Izidoro Blikstein. 25 ed. São Paulo: Cultrix, 1996.

Curso de lingüística geral. Organização de Charles Bally e Albert Sechehaye com a colaboração de Albert Riedlinger. Trad. de Antônio Chelini, José Paulo Paes e Izidoro Blikstein. 24 a ed. São Paulo: Pensamento-Cultrix, 2002.

TAYLOR, René. El Arte De La Memoria En El Nuevo Mundo. Madrid: editorial Swan colonia inglesa, 1987.

VYGOTSKY, L.S. Pensamento e Linguagem. São Paulo: Martins Fontes, 1989.

YATES, Frances. A Arte Da Memoria. Campinas, São Paulo: Editora da Unicamp, 2007 